\title{
Noise-driven amplification mechanisms governing the emergence of coherent extreme events in excitable systems
}

\author{
Lluís Hernández-Navarro $\odot,{ }^{1, *}$ Sergio Faci-Lázaro $\odot,{ }^{2,3}$ Javier G. Orlandi $\odot,{ }^{4}$ Ulrike Feudel $\odot,{ }^{5}$ \\ Jesús Gómez-Gardeñes $\mathbb{C}^{2,3}$ and Jordi Soriano ${ }^{6,7, \dagger}$ \\ ${ }^{1}$ Institut d'Investigacions Biomèdiques August Pi i Sunyer (IDIBAPS), Barcelona 08036, Spain \\ ${ }^{2}$ Department of Condensed Matter Physics, University of Zaragoza, Zaragoza 50009, Spain \\ ${ }^{3}$ GOTHAM Laboratory, Institute of Biocomputation and Physics of Complex Systems (BIFI), University of Zaragoza, Zaragoza 50018, Spain \\ ${ }^{4}$ RIKEN Center for Brain Science, Wako-shi, 351-0198 Saitama, Japan \\ ${ }^{5}$ Institute for Chemistry and Biology of the Marine Environment, Carl von Ossietzky University Oldenburg, 26111 Oldenburg, Germany \\ ${ }^{6}$ Departament de Física de la Matèria Condensada, Universitat de Barcelona, Barcelona 08028, Spain \\ ${ }^{7}$ Universitat de Barcelona Institute of Complex Systems (UBICS), 08028 Barcelona, Spain
}

(Received 17 November 2020; accepted 16 April 2021; published 21 May 2021)

\begin{abstract}
The physics governing the formation of extreme coherent events, i.e., the systemwide emergence of an observable taking extraordinary values in a short time window, is a relevant yet elusive problem to a variety of disciplines ranging from climate science to neuroscience. Despite their inherent differences, systems exhibiting episodes of extreme coherence can be abstracted as a set of coupled nonlinear elements in a noisy and networked environment. Here, we propose a model describing the generation of extreme coherence by exploring theoretically and numerically the capacity of noise and network correlations to amplify a critical core of the system and trigger an extreme event. Although we principally center our study in modeling bursting phenomena in neuronal circuits, we extend our analysis to other systems such as algae blooms and infectious diseases. We show that extreme events originate in a relatively small core of the system and that different cores may coexist. We also show that the amplification mechanisms within a system are highly robust, so that the deletion of central nodes leads to other nodes taking leadership.
\end{abstract}

DOI: 10.1103/PhysRevResearch.3.023133

\section{INTRODUCTION}

The emergence of recurrent but rare events characterized by a certain observable taking on utmost values compared with the usual behavior can often be considered as an extreme event. There are ample examples of this type of event in nature [1-3], such as harmful algal blooms, where a species usually present at low densities all of a sudden becomes extremely abundant and dominant in a plankton community [4,5]; the outbreak of an infectious disease, where the number of infected individuals explodes over a very short time [6]; neuronal avalanches in cortical tissue [7,8]; epileptic seizures, in which a very intense and synchronous neuronal activity pattern emerges in a patch of brain tissue [9]; or rogue waves occurring in the ocean [10-12] or in laser systems [13-15].

Several mechanisms have been discovered regarding how such extreme events could be created. They include "interior

\footnotetext{
*1luishn@gmail.com

†jordi.soriano@ub.edu

Published by the American Physical Society under the terms of the Creative Commons Attribution 4.0 International license. Further distribution of this work must maintain attribution to the author(s) and the published article's title, journal citation, and DOI.
}

crises" [16-18], Pomeau-Manneville intermittency [19], the quasiperiodicity route to chaos [20], or attractor hopping in multistable systems [21,22]. For example, the various mechanisms to create rogue waves $[23,24]$ in the ocean are explained in terms of Peregrine solitons [25], whereas some of the studies on extreme events on networks employ excitable units operating in an oscillatory mode on each node to demonstrate the emergence of extreme events, either as stable dynamics [16] or as part of unstable transient dynamics [26].

In the context of neuroscience, the capacity of neuronal circuits to exhibit extreme events has been investigated in an attempt to understand the mechanisms leading to epileptic seizures [27] and the bursting phenomenon, in which the whole neuronal network - or a large fraction of it-activates coherently in a short time window, e.g., as occurs in neuronal cultures [28-30]. It must be noted, however, that extreme events could be viewed in a similar way as bursting episodes in a neuronal network since they partly consist of bursts in the whole neuron community, which are recurrent but still rare, with very large interburst intervals (IBIs). As soon as they become more frequent, or even almost periodic, they would no longer fulfill the condition of rareness and could not be considered extreme events.

Modeling scenarios for epilepsy and sudden bursting extend from the microscale, in the form of detailed representations of neurons coupled through complex topologies, to the macroscale, with the introduction of neural mass models or 
oscillators that represent brain regions coupled to one another in a nonlinear way. Despite the importance of these models, an aspect that has not been explored in detail is the role of noise and connectivity correlations in triggering sudden networkwide activity.

In this paper, we take a mesoscale approach to understand the emergence of sudden bursting and analyze in detail the activity amplification mechanisms, grounded on noise and connectivity correlations, that occur in a small region of a neuronal circuit that we term core. We derive the simplest theoretical model able to capture burst initiation dynamics, providing a deep insight into the necessary ingredients for the emergence of extreme coherent events in a neuronlike excitable system. Once a burst is generated, its spatiotemporal structure and termination mechanisms strongly depend on both the nature of the excitable system and the topological details of the network in which it is embedded, effectively shaping a completely different dynamic scenario. Thus, for sake of clarity and simplicity, in this paper, we focus only on burst initiation.
Firstly, we analyze analytically and numerically the conditions that have to be met, in terms of noise and the topological details of a network, for an avalanche of amplification to succeed and trigger a burst. Secondly, we explore the resilience of burst initiation to a directed attack on neurons that exhibit central characteristics. Thirdly, we investigate the role that noise and topological amplification mechanisms play to trigger extreme events in other natural systems that share the same ingredients, i.e., inherently noisy nonlinear elements coupled through a complex network.

\section{FRAMEWORK: NOISE FOCUSING AND ACTIVITY AMPLIFICATION MECHANISMS}

The "noise focusing" framework is based on the concept that random neuronal activations are amplified by both metric correlations and noise [30]. Metric correlations are topological features that emerge from the spatial embedding of neurons and the finite length of axons and dendrites. These features include local connectivity and high clustering (a) no amplification

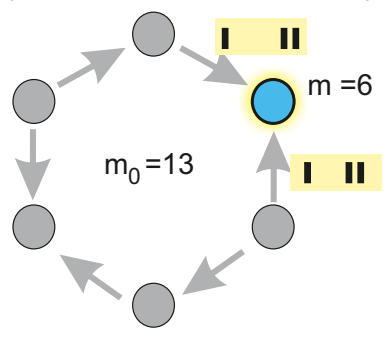

(e)

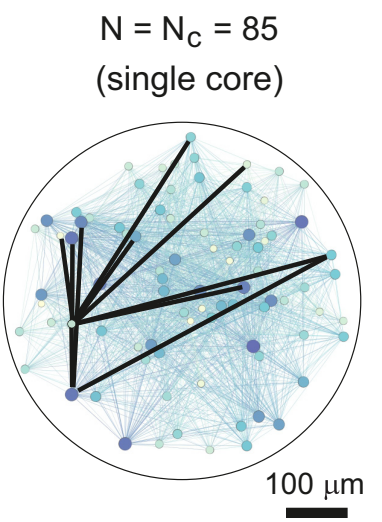

(b) topological amplification

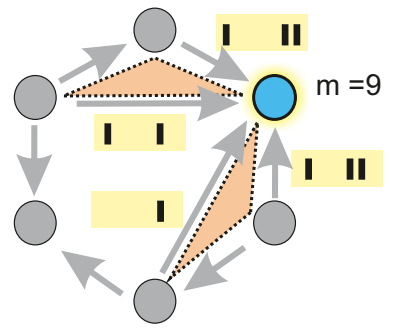

(c)

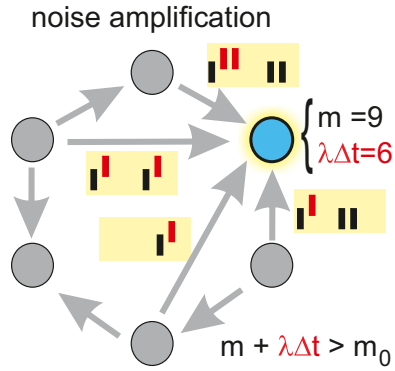

(d)

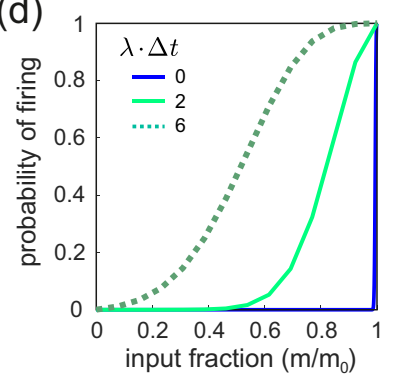

(f)

$\mathrm{N}=2000$ (multiple cores)

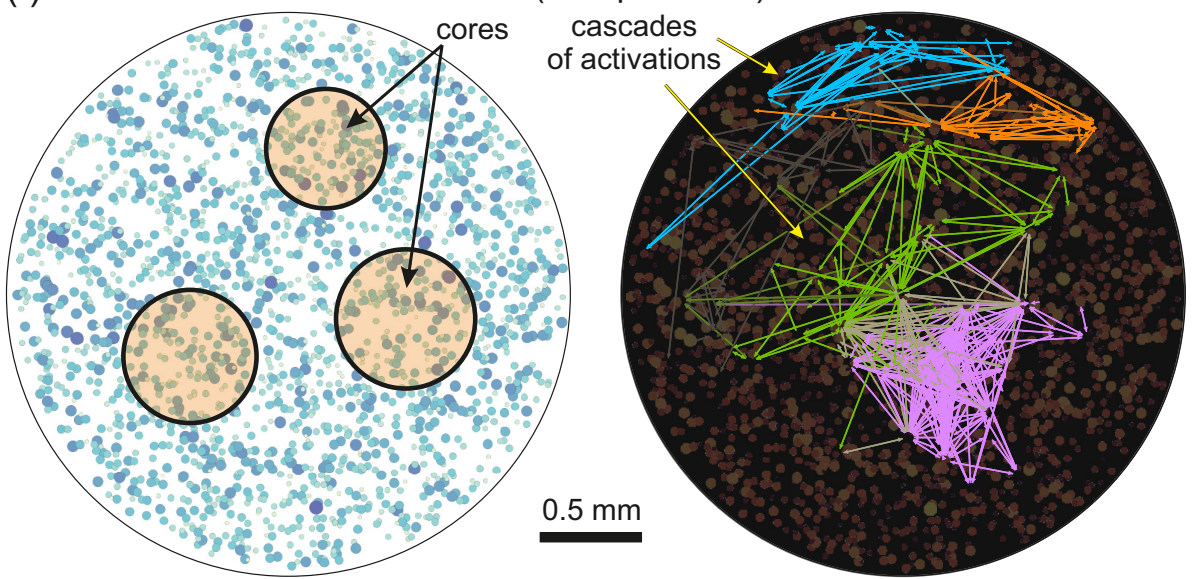

FIG. 1. Dynamic amplification mechanisms and concept of core. (a) In the absence of amplification, a target neuron (blue) needs $m_{0} \simeq 13$ inputs (black bars) in $\Delta t \simeq 20 \mathrm{~ms}$ (yellow box) to activate. (b) Topological amplification arising from loops in the network (orange) increases the number of inputs onto the neuron, facilitating quorum. (c) The presence of noise (red bars) further increases the effective number of inputs onto the target neuron, which now satisfies the quorum condition. (d) Neuronal firing probability as a function of $m / m_{0}$ for different noise strengths $\lambda \Delta t$, showing the substantial increase in firing probability for even weak noise amplification. Here, $\lambda$ is the mean frequency of inputs generated by synaptic noise. (e) Numerical realization of a neuronal network to illustrate the concept of core, which is the minimum ensemble of neurons (typically $N=N_{C} \simeq 85$ ) in a network able to generate a burst. Color dots and lines are neurons and connections, respectively. The diameter of the neurons and the strength of the color is proportional to their connectivity. Black lines show a cascade of activations (avalanche), although not sufficiently strong to generate a burst. (f) Left, Numerical realization of a network with $N=2000$ neurons, showing three representative cores of radius $r_{c}=300 \mu \mathrm{m}$. Connections are omitted for clarity. Right, Examples of avalanches in the network. Colors depict independent spatiotemporal events. 
coefficients since physically close neurons are more likely to connect and promote the existence of feedforward and feedbackward loops [30-32]. Noise, on the other hand, originates from both fluctuations in the membrane potential and the spontaneous release of neurotransmitters in the synaptic boutons of presynaptic neurons [30].

Activity amplification is grounded on the nonlinear, integrate-and-fire nature of neurons. In the noise focusing framework, this central characteristic is formulated as the need for quorum, i.e., the arrival of a sufficient number of inputs into the membrane for it to pass the threshold and activate [33]. As sketched in Fig. 1(a), a neuron is activated whenever it receives at least $m_{0}$ inputs from its neighbors in a time window of $\Delta t=20 \mathrm{~ms}$ [33-36]. Typically, $m_{0} \simeq 13$ in neuronal cultures $[33,35]$. The existence of loops and recurrent activity, promoted by metric correlations [Fig. 1(b)], raises overall neighboring activity and leads to a higher cascade of neuronal activations. Similarly, noise adds new activations to the train of spikes converging onto a neuron [Fig. 1(c)], substantially increasing its probability of firing [Fig. 1(d)].

Both amplification mechanisms, as delineated by Orlandi et al. [30], suffice to activate a small but critical core of the network of typically $r_{c} \simeq 300 \mu \mathrm{m}$ in radius and containing about $N_{c} \sim 85$ neurons [30] [Fig. 1(e)]. A core has to be understood as any ensemble of neurons large enough to independently generate an avalanche of activity (a cascade of concatenated neuronal activations) and ignite the whole network, shaping a network burst or extreme event. Multiple cores may therefore exist in large networks containing thousands of neurons [Fig. 1(f)], thus increasing the probability for a burst to occur. Once a burst is initiated, its spatiotemporal propagation depends on the connectivity details of the neuronal circuit, ranging from fast circular fronts in homogeneous networks to intricate spatiotemporal waves in heterogeneous or engineered circuits [37].

\section{THEORETICAL MODEL}

In the following, we will consider a neuronal network constituted by only excitatory neurons, which allows us to obtain analytical solutions. The impact of inhibition in the model is discussed later. We will also consider that, once a given neuron has satisfied the quorum condition of $m_{0}$ total inputs in $\Delta t=20 \mathrm{~ms}$, it will activate and pass the signal to its neighbors in the following time window of $\delta t \sim 2-3 \mathrm{~ms}$. This time delay captures the biophysical processes associated with action potentials and the generation of postsynaptic currents and is important to derive realistic time scales for burst initiation. Table I summarizes the parameters used by the model and its predictions.

\section{A. Single firing probability equation}

The subquorum probability of firing is modeled as a Poisson shot process in the membrane potential, with spontaneous "shot noise" mean frequency $\lambda$, in a time window $\Delta t$, and where $m$ positive inputs from active neuron neighbors would contribute as single, nonnoise shots $[30,38]$. Biologically, the
TABLE I. Summary of the parameters used and their values for the theoretical model and numerical simulations. The last five values are predictions of the model. Std $=$ standard deviation, Prob. $=$ probability.

\begin{tabular}{lcc}
\hline \hline Notation & Numerical value & Description \\
\hline BA/IA & - & Background/ignition avalanche \\
$N$ & $\sim 5 \times 10^{4}$ neurons & Total neurons \\
$N_{c}$ & $\approx 85$ neurons & Total neurons in critical subset \\
$r_{c}$ & $\sim 300 \mu \mathrm{m}$ & Radius of critical subset \\
$k \equiv k_{N_{c}}^{\text {in }}$ & $\sim 30-40$ neurons & Average intra- $N_{c}$ connectivity \\
$\Delta t$ & $20 \mathrm{~ms}$ & Time window for inputs \\
$\delta t$ & $2-3 \mathrm{~ms}$ & Time step in avalanches \\
$\tau_{\text {refrac }}$ & $\sim 10 \mathrm{~s}$ & Culture recovery time \\
$\omega_{0}$ & $\sim 0.1 \mathrm{~Hz} /$ neuron & Spontaneous activation rate \\
$\lambda$ & $\approx 300 \mathrm{~Hz} /$ neuron & Shot noise rate \\
$m_{0}$ & 13 & Initial demand of inputs \\
$n$ & - & Active neurons in $N_{c}$ and $\Delta t$ \\
$m$ & - & Inputs from active neighbors \\
$\mu_{\text {shot }}$ & $\approx 7.5$ & $\langle$ min $(m)\rangle$ to fire \\
$\sigma_{\text {shot }}$ & $\approx 2.2$ & Std of min $(m)$ to fire \\
$\mu_{\text {hyp }}$ & $($ depends on $n)$ & $\langle m\rangle$ in $N_{c}$ and $\Delta t$ \\
$\sigma_{\text {hyp }}$ & $($ depends on $n)$ & Std of $m$ in $N_{c}$ and $\Delta t$ \\
$\Delta n$ & $($ depends on $n)$ & Single step mean increase in $n$ \\
$\sigma_{\Delta n}$ & $($ depends on $n)$ & Single step std of increase in $n$ \\
$n_{\text {th }}$ & $4.7 \pm 1.0$ & Threshold in $n$ for BA/IA \\
$p_{\text {subburst }}^{\Delta t}$ & $\approx 3 \times 10^{-6}$ & Prob. of $N_{c}$ activation in $\Delta t$ \\
$p_{\text {burst }}^{\Delta t}$ & $\approx 1.7 \times 10^{-3}$ & Prob. of culture burst in $\Delta t$ \\
$\langle I B I\rangle$ & $\sim 21 \mathrm{~s}$ & Mean interburst interval \\
$\nu_{\text {burst }}$ & $\sim 4.7 \times 10^{-2}$ & Culture bursting frequency \\
\hline \hline & &
\end{tabular}

former shot noise corresponds to spontaneous miniature excitatory postsynaptic currents (mEPSCs).

The probability $q \equiv p\left(\uparrow \mid m, m_{0}, \lambda \Delta t\right)$ of a neuron to activate ( $\uparrow)$, with $m$ inputs from neighbors in a time window $\Delta t$ and a quorum $m_{0}$, is thus the probability of spontaneously receiving $m_{0}-m$ (or more) total noise shot inputs, with an average of $\lambda \Delta t$. This can be expressed as

$$
q=e^{-\lambda \Delta t} \sum_{i=m_{0}-m}^{\infty} \frac{(\lambda \Delta t)^{i}}{i !}=1-\mathcal{P}\left(m_{0}-m, \lambda \Delta t\right),
$$

where $\mathcal{P}$ is the regularized $\Gamma$ function, $m_{0}$ is the total demand of inputs, and $m$ is the effective number of active neighbors' inputs in the time window $\Delta t$.

The Poisson shot process can be approximated by a normal distributed shot process with mean $\lambda \Delta t+\frac{1}{2}$ and standard deviation $\sqrt{\lambda \Delta t}$. Equation (1) can then be written in a compact manner as

$$
q \simeq 1-\Phi\left[\frac{\left(m_{0}-m\right)-\left(\lambda \Delta t+\frac{1}{2}\right)}{\sqrt{\lambda \Delta t}}\right],
$$

where $\Phi$ is the standard normal cumulative distribution function. By introducing $\mu_{\text {shot }} \equiv m_{0}-\left(\lambda \Delta t+\frac{1}{2}\right)$ and $\sigma_{\text {shot }} \equiv$ $\sqrt{\lambda \Delta t}$, we obtain

$$
q=\Phi\left(\frac{m-\mu_{\text {shot }}}{\sigma_{\text {shot }}}\right)
$$


which describes the probability that a neuron will fire in the next time step $\delta t$ upon receiving inputs from $m$ active neighbors within the previous time window $\Delta t$.

\section{B. Single neuron active inputs within the $N_{c}$ core}

Bursts initiate as a result of the topological amplification of noise and neuronal activity, which ignites a critical subset of size $N_{c}$ neurons. Topologically, $N_{c}$ can be approximated as a random graph since its characteristic diameter of $2 r_{c}=600 \mu \mathrm{m}$ is shorter than the average axonal length of $\simeq 1 \mathrm{~mm} \mathrm{[32].}$

We term avalanche as any cascade of activity involving connected neurons and with $<50 \mathrm{~ms}$ of time difference between activations. Larger times indicate the beginning of a new avalanche. This definition is often referred to as "causal avalanche," in the sense that it is not sufficient for any two neurons to activate within the same time bin; one has to drive the other through an existing connection. Illustrative examples of causal avalanches are shown in Figs. 1(e) and 1(f).

We denote by $n$ the ensemble of spontaneously active neurons on $N_{c}$ in an initial time window $\Delta t=20 \mathrm{~ms}$. A burst will occur whenever the activity-amplification mechanism leads to $n \simeq N_{c}$ on the core network. Thus, there is initially a population of $N_{c}-n$ neurons that are inactive in the first $\Delta t$ time window.

For a given neuron, its probability to fire will depend on its connectivity $k$, which determines the probability $r \equiv$ $p\left(m \mid n, k, N_{c}\right)$ to receive $m$ inputs from $n$ active neighbors among all $N_{c}-1$ possible neighbors. If the input connectivity is fixed for all neurons in the core network as $k \equiv k_{N_{c}}^{\text {in }}$, the total number of possible combinations of $m$ active inputs is then given by

$$
\left(\begin{array}{c}
N_{c}-1-n \\
k-m
\end{array}\right)
$$

Since neurons are distinguishable, all possible combinations of $m$ elements over $n$ have to be considered, i.e., $\left(\begin{array}{l}n \\ m\end{array}\right)$, for each combination in Eq. (4). The final normalized probability distribution function $r$ then reads

$$
r=\frac{\left(\begin{array}{c}
n \\
m
\end{array}\right)\left(\begin{array}{c}
N_{c}-1-n \\
k-m
\end{array}\right)}{\left(\begin{array}{c}
N_{c}-1 \\
k
\end{array}\right)}
$$

This equation can be identified with the hypergeometric distribution, where the number of successes corresponds to $m$, in $k$ draws without replacement, from a population of $N_{c}-1$ that contains $n$ objects with the desired feature. This facilitates an analytical exploration of Eq. (5) by approximating the hypergeometric function to a normal one with mean $\mu_{\text {hyp }}$ and standard deviation $\sigma_{\text {hyp }}$ given by

$$
\begin{gathered}
\mu_{\text {hyp }}=n \frac{k}{N_{c}-1}, \\
\sigma_{\text {hyp }}=\sqrt{\frac{1}{N_{c}-2} n\left(1-\frac{n}{N_{c}-1}\right) k\left(1-\frac{k}{N_{c}-1}\right)} .
\end{gathered}
$$

The $r$ distribution then reads

$$
r=\frac{1}{\sigma_{\text {hyp }}} \varphi\left(\frac{m-\mu_{\text {hyp }}}{\sigma}\right),
$$

where $\varphi(x)$ is the standard normal density function with zero mean and one standard deviation.

\section{Probability of next-step activation}

At this point, two central ingredients have been articulated. On the one hand, Eq. (3) characterizes the probability of activation of an arbitrary, inactive neuron when $m$ of its input neighbors are active in a time window of $\Delta t=20 \mathrm{~ms}$. On the other hand, Eq. (8) describes the probability for a neuron to be connected to $m$ active input neighbors when there exist $n$ active neurons in a core $N_{c}$ with average connectivity $k$.

The combination of both probabilities allows us to determine $p \equiv p\left(\uparrow \mid m_{0}, \lambda \Delta t, n, k, N_{c}\right)$ the activation probability of an arbitrary, inactive neuron depending directly on the initial number of active neurons $n$ in the subset $N_{c}$. This can be expressed as the average dynamic correlations marginalized over $m$, i.e.,

$$
p=\sum_{m=0}^{n} q r
$$

which from Eqs. (3) and (8) can be approximated to

$$
p \simeq \int_{\frac{1}{2}}^{n+\frac{1}{2}} \frac{\Phi\left(\frac{m-\mu_{\text {shot }}}{\sigma}\right) \varphi\left(\frac{m-\mu_{\text {hyp }}}{\sigma_{\text {hyp }}}\right)}{\sigma_{\text {hyp }}} d m .
$$

By redefining $x \equiv \frac{m-\mu_{\text {hyp }}}{\sigma_{\text {hyp }}}$ and $d x \equiv \frac{d m}{\sigma_{\text {hyp }}}$, we obtain

$$
p \simeq \int_{\frac{\frac{1}{2}-\mu_{\text {hyp }}}{\sigma_{\text {hyp }}}}^{\frac{\frac{1}{2}+n-\mu_{\text {hyp }}}{\sigma_{\text {hyp }}}} \Phi(a+b x) \varphi(x) d x,
$$

where $a \equiv \frac{\mu_{\text {hyp }}-\mu_{\text {shot }}}{\sigma_{\text {shot }}}$ and $b \equiv \frac{\sigma_{\text {hyp }}}{\sigma_{\text {shot }}}$.

The argument of the last integral is like a Gaussian whose main area falls within the integration limits, particularly for $n \gtrsim 2$. Thus, we can approximate the upper and lower integral bounds of Eq. (11) by $+\infty$ and $-\infty$, respectively. Using Ref. [39],

$$
\int_{-\infty}^{+\infty} \Phi(a+b x) \varphi(x) d x=\Phi\left(\frac{a}{\sqrt{1+b^{2}}}\right),
$$

we finally get

$$
\begin{aligned}
p & \simeq \Phi\left[\frac{\mu_{\mathrm{hyp}}(n)-\mu_{\mathrm{shot}}}{\sqrt{\sigma_{\mathrm{hyp}}(n)^{2}+\sigma_{\mathrm{shot}}^{2}}}\right]= \\
& =\Phi\left\{\frac{n \frac{k}{N_{c}-1}-\left[m_{0}-\left(\lambda \Delta t+\frac{1}{2}\right)\right]}{\sqrt{\frac{n\left(1-\frac{n}{N_{c}-1}\right) k\left(1-\frac{k}{N_{c}-1}\right)}{N_{c}-2}+\lambda \Delta t}}\right\} .
\end{aligned}
$$

This equation sets the probability that the number of inputs for an arbitrary neuron is larger or equal to what it needs to reach $m_{0}$ threshold, with shot noise and network activity already considered. Hence, Eq. (13) captures the probability of activation of a neuron within $N_{c}$ in a time window of $\delta t=2-3 \mathrm{~ms}$, provided that $n$ neurons activated in the previous $\Delta t=20 \mathrm{~ms}$ window. 


\section{Dynamical difference equation for the $N_{c}$ core}

To complete the description of the $N_{c}$ core dynamics, an evaluation of the inactivation rate of neurons is required. We note that a moving time window $\Delta t$ would gradually lose (or attenuate) the contribution of the oldest activations at an average initial rate of $n / \Delta t$. Thus, since our time step is set at $\delta t=2-3 \mathrm{~ms}$ due to the time duration of action potentials, the number of activations whose effect is lost at the next time step is, on average, $\Delta n^{-}=\delta t n / \Delta t$.

By using Eq. (13), and noting that the available number of neurons to activate is $N_{c}-n$, the number of inactive neurons that on average would become active at the next time step is $\Delta n^{+}=p(n)\left(N_{c}-n\right)$. Both the activation and the inactivation rates follow Poisson distributions, with their corresponding means. The evolution of $n$ is then governed by the difference of two Poisson-distributed variables, whose resulting distribution is the Skellam distribution, of mean $\Delta n^{+}-\Delta n^{-}$and standard deviation $\sqrt{\Delta n^{+}+\Delta n^{-}}$. Therefore, the dynamical first-order difference equation of the $N_{c}$ core network is

$$
n_{t+\delta t}=n_{t}+p\left(n_{t}\right)\left(N_{c}-n_{t}\right)-\frac{\delta t}{\Delta t} n_{t},
$$

which explicitly reads

$$
\begin{aligned}
\Delta n= & \Phi\left\{\frac{n \frac{k}{N_{c}-1}-\left[m_{0}-\left(\lambda \Delta t+\frac{1}{2}\right)\right]}{\sqrt{\frac{n\left(1-\frac{n}{N_{c}-1}\right) k\left(1-\frac{k}{N_{c}-1}\right)}{N_{c}}+\lambda \Delta t}}\right\} \\
& \times\left(N_{c}-n\right)-\frac{\delta t}{\Delta t} n,
\end{aligned}
$$

where $\Delta n \equiv n_{t+\delta t}-n_{t}$ is the forward finite difference of $n$ at time $t$, and where we dropped the subindex $t$ for clarity.

\section{E. Threshold for ignition avalanches}

Equation (15) describes the capacity of $n$ initially active neurons to amplify activity and ignite the $N_{c}$ core. An initial ensemble $n$ that is too small would lead to avalanches that would die out [background avalanches (BA)] except for spontaneous activity, whereas an $n$ sufficiently large would ensure enough amplification for the entire core to light up and start a burst [ignition avalanches (IA)]. The threshold value $n_{\text {th }}$ that separates BA from IA can be viewed as an unstable equilibrium point, in the sense that any perturbation in $n$ would drag the system either toward BA or IA.

Figure 2(a) shows a representative numerical realization of Eq. (15) with biophysically plausible parameter values that provide $n_{\text {th }} \simeq 5$, as described later. Positive values for the next-step increment $\Delta n$ indicate a growth in network activity, whereas negative values depict a decrease in neuronal activations. A zero increment indicates an equilibrium state of the system. A detail of the dynamical process is shown as a cobweb plot in Fig. 2(b). A BA (red) swiftly vanishes at $n \simeq 4$, while an IA (green) keeps growing until it encompasses the entire core. The steplike profile of the IAs indicates that the number of new neurons participating in the avalanche process quickly grows as amplification progresses.

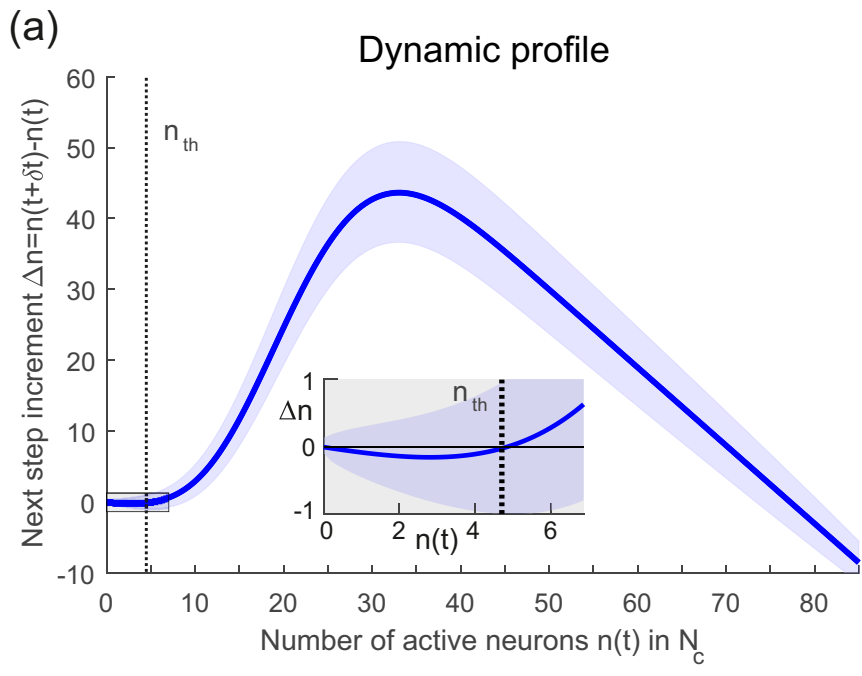

(b)

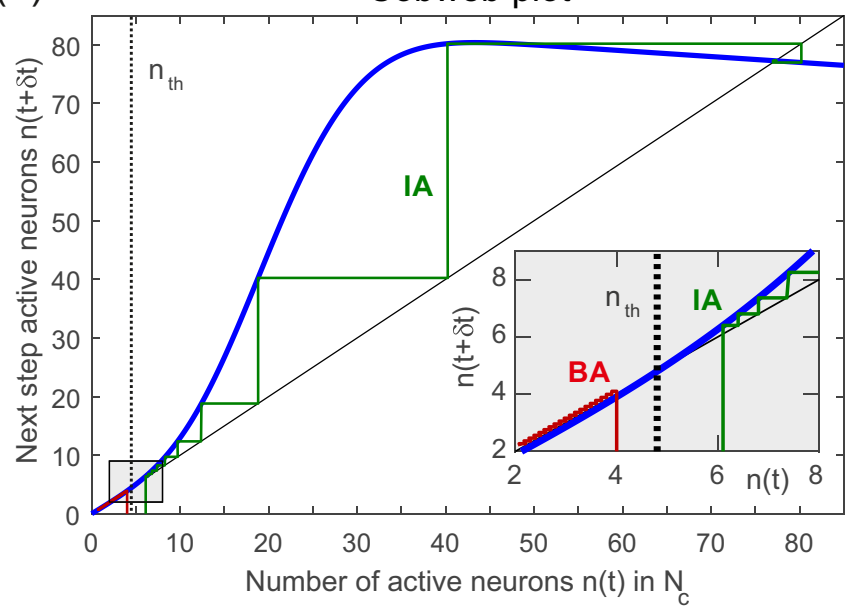

FIG. 2. Dynamic amplification process and avalanches. (a) The thick blue line depicts the increment in the number of active neurons $\Delta n$ in the following time step $t+\delta t$ as a function of the number of active neurons $n$ in current time step $t$. The blue shading provides the upper and lower bounds for the Skellam distribution. Inset: Detail of the neighborhood around the threshold value $n_{\text {th }}$ (black dashed line) that separates background avalanches (BAs) from ignition avalanches (IAs). (b) Cobweb plot illustrating the cascade of neuronal activity within the $N_{c}$ core, with example trajectories for a BA (red) and an IA (green). Inset: Detail of the dynamic evolution in the vicinity of the threshold region.

The amplification process can be viewed as a potential in the context of classical mechanics. We term it increment potential, and it is numerically calculated as $-\int_{0}^{n} \Delta n d n$.

As shown in Fig. 3, and similarly to classical mechanics, a minimum in the potential depicts a stable state, while a maximum value indicates an unstable equilibrium. When no bursting activity is present, critical $N_{c}$ subsets dwell in the leftmost, stable region of BAs. At some point, the dynamical and topological amplification of noise increases the activity of the network beyond the unstable equilibrium, enforcing an IA and dragging the system to a stable bursting regime.

The evaluation of the threshold value $n_{\text {th }}$ introduced above was carried out by determining those states that compensated 


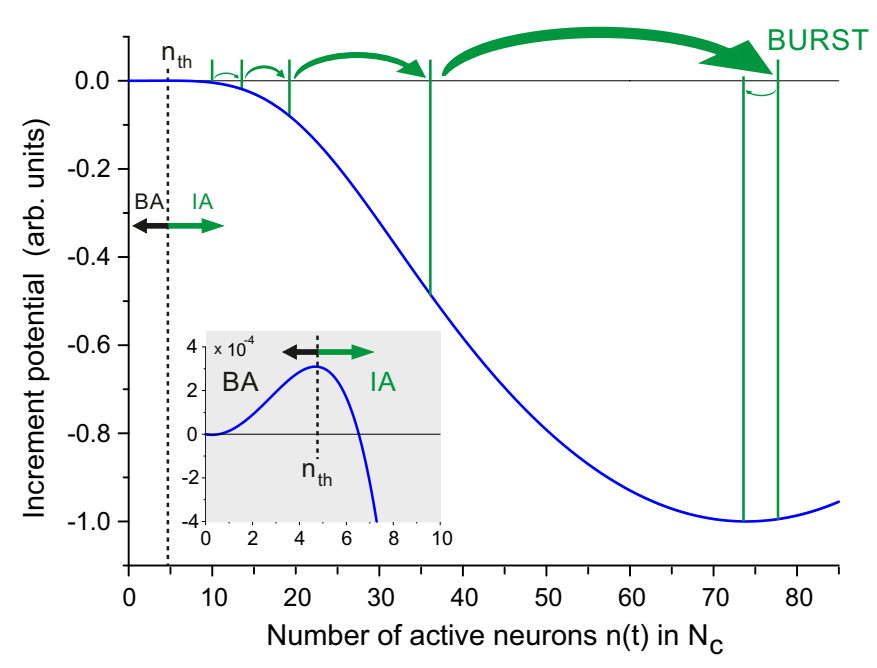

FIG. 3. Increment potential. Illustrative approximate parallelism to the potential concept in classical mechanics. Solid blue line shows $-\int_{0}^{n} \Delta n d n$ derived numerically from the data of Fig. 2 . The vertical dashed black line shows the threshold value $n_{\text {th }}$ that separates background avalanches (BAs) and ignition avalanches (IAs). The vertical green lines and arrows represent the dynamical evolution of the system for the IA of Fig. 2. Inset: Detail of the unstable equilibrium point at the vicinity of the threshold $n_{\mathrm{th}}$.

average activations and average attenuations, i.e., zero mean rates or zero derivative states. Indeed, from Eq. (15), the equilibrium states of $n$ are solutions of the equation

$$
p(n)\left(N_{c}-n\right)-\frac{n}{\Delta t} \delta t=0,
$$

and by using $N_{c} \sim 85$ neurons, $k \sim 30$ input neurons over $N_{c}$, $m_{0} \simeq 13$, and $\delta t=3 \mathrm{~ms}$, a family of solutions at equilibrium $n_{\text {eq }}$ can be determined, procuring $n_{\text {eq }}=\{0.28,4.69,73.9\}$ active neurons. The first solution is stable and corresponds to background activity. The third solution is also stable and is related to peak bursting activity, i.e., the presence of strong activity in the network. The second solution is unstable and corresponds to our solution of interest, i.e., the threshold $n_{\text {th }}$ that separates BAs from IAs.

It is important to note that the third solution is kinetically stable, meaning that it exists as far as the network is able to maintain high levels of activity. Such a scenario holds only at short time scales from a biophysical perspective since sustained activity eventually leads to the depletion of neurotransmitters and to a refractory period of no activity. For simplicity, and since this paper focuses on initiation rather than propagation of bursting activity, the theoretical model does not consider this short-term depression mechanism. We also note that peak bursting activity does not encompass the isolated $N_{c}$ core but neighboring regions as well and that high levels of activity can trigger a burst in surrounding cores. These multicore dynamic interactions go beyond the scope of this paper and were already treated by Orlandi et al. [30].

An estimation of the uncertainty associated with the solution of our interest $n_{\text {th }} \equiv n_{\text {eq }} \simeq 4.7$ can be carried out by using the Skellam's standard deviation, which provides $\sigma=\sqrt{\Delta n_{\mathrm{eq}}^{+}+\Delta n_{\mathrm{eq}}^{-}}=\sqrt{2 \Delta n_{\mathrm{eq}}^{-}}=\sqrt{2 \delta t n_{\mathrm{eq}} / \Delta t}$. We thus conclude that the threshold for the generation of IAs is given by

$$
n_{\mathrm{th}} \simeq 4.7 \pm 1.0
$$

\section{F. Whole-culture bursting: Ensemble of $N / N_{c}$ independent subnetworks}

Real neuronal networks contain a number of neurons $N$ that is much larger than $N_{c}$. Thus, in the context of our model, one could expect $\sim N / N_{c}$ nonoverlapping cores able to generate bursting episodes that would propagate throughout the entire network. We note that, from an analytical perspective, the concept of core facilitates a partition of the network into local and global connectivity scales. Since the local scale is on the order of the diameter of the typical dendritic tree ( $\simeq 600 \mu \mathrm{m}$ ) and shorter than the characteristic axonal length ( $\simeq 1 \mathrm{~mm}$ ), any core can be effectively modeled as a random graph, with all cores exhibiting an equivalent connectivity. This approach greatly simplifies the analytical derivations.

Thus, if all cores exhibit similar characteristics, the probability of activation of an arbitrary core in $\Delta t$ can be calculated as the probability of spontaneous occurrence of $n \geqslant n_{\text {th }}$, with $n$ Poisson-distributed, i.e.,

$$
p_{\text {subburst }}^{\Delta t}=1-\mathcal{P}\left(n_{\mathrm{th}}, \omega_{0} \Delta t N_{c}\right),
$$

where $\mathcal{P}$ is, again, the regularized $\Gamma$ function and $\omega_{0} \Delta t N_{c}$ the mean of the Poissonian process. We take $\omega_{0}=0.1 \mathrm{~Hz}$ as the characteristic neuronal spontaneous activation frequency $[30,40]$.

Since at least one core has to activate during a $\Delta t$ window to observe a whole-network burst, the bursting probability in $\Delta t$ is then given by

$$
p_{\text {burst }}^{\Delta t}=1-\left(1-p_{\text {subburst }}^{\Delta t}\right)^{\frac{N}{N_{c}}}=1-\left[\mathcal{P}\left(n_{\text {th }}, \omega_{0} \Delta t N_{c}\right)\right]^{\frac{N}{N_{c}}} .
$$

The average IBI, i.e., the time span between two consecutive bursting episodes, can be evaluated by considering the recovery time $\tau_{\text {rec }}$ that has to elapse for cultures to burst again. This recovery time is associated with burst termination and depends on a number of factors, including short-term synaptic depression (i.e., the gradual depletion of neurotransmitters), action potential hyperpolarization, and the amount of inhibition in the network. For simplicity, and since burst termination mechanisms are not included in the model, we use $\tau_{\text {rec }}=10 \mathrm{~s}$, which is the minimum characteristic time scale for burst recovery observed in neuronal cultures similar to the modeled ones [41]. The average IBI is then

$$
\langle\mathrm{IBI}\rangle=\tau_{\mathrm{rec}}+\frac{\Delta t}{p_{\text {burst }}^{\Delta t}}=\tau_{\mathrm{rec}}+\frac{\Delta t}{1-\left[\mathcal{P}\left(n_{\mathrm{th}}, \omega_{0} \Delta t N_{c}\right)\right]^{\frac{N}{N_{c}}}} \sim 21 s,
$$

where we have taken $N=5 \times 10^{4}$ neurons as in typical experimental preparations with neurons homogeneously distributed over a flat surface [41]. The bursting frequency is then $\nu_{\text {bursts }}=$ $1 /\langle\mathrm{IBI}\rangle \simeq 3$ bursts $/ \mathrm{min}$.

We note that the uncertainty in the value of $n_{\text {th }}$ in Eq. (17) provides an order of magnitude of unpredictability in the IBIs, between $\sim 11$ and $\sim 350 \mathrm{~s}$. This broad range is consistent with experimental observations, which reported IBIs in the range 
10-200 s [41-43] and that strongly depended on the developmental stage of the neuronal networks and their biochemical environment. Thus, the model can capture well the mechanisms governing burst initiation and the time scales involved but cannot precisely infer IBIs since they are highly sensitive to the dynamical traits of the neurons and the topological characteristics of the network.

\section{NUMERICAL RESULTS}

We carried out numerical simulations of neuronal networks to gain further insight on the mechanisms governing burst initiation. Following Refs. [30,44], we considered spatial in silico networks that were set on a two-dimensional surface, with neurons homogeneously distributed and connecting to one another following biologically realistic axonal growth rules. The main ingredients of the network construction were

(i) Neuronal cell bodies with fixed diameter $\phi_{s}$ randomly positioned over the surface.

(ii) A dendritic tree for each neuron, centered at its soma, and extending a diameter $\phi_{d} \simeq 600 \mu \mathrm{m}$ given by a Gaussian distribution with average $\mu_{d}$ and standard deviation $\sigma_{d}$.

(iii) An axon departing from the soma of each neuron with a total length $l$ given by a Rayleigh distribution with standard deviation $\sigma_{l}$. Axons were laid out as concatenated segments $\Delta l$. The orientation of each segment followed a Gaussian distribution with standard deviation $\sigma_{\theta}$ and centered around the previous segment. The first segment was oriented randomly relative to the soma.

(iV) A connection was established whenever the axon of a neuron intersected the dendritic tree of another neuron and according to a probability $\alpha=0.1$. This probability was independent of the overlapping length between the axon and the dendritic tree.

Examples of the generated networks are provided in Figs. 1(e) and 1(f). This biologically realistic construction of the in silico network shaped metric correlations in the form of high-clustering coefficients and loops, which facilitated activity amplification. However, at the spatial scales of the dendritic tree (600 $\mu \mathrm{m}$ diameter) a neuron could effectively connect with any other within the neighborhood $[32,45]$. Thus, connectivity at this scale can be viewed as a random graph with small fluctuations inherited from metric correlations.

The Izhikevich model [46] was used to simulate the behavior of the membrane potential of the neuron and synapses. Following the implementation of Orlandi et al. [30], the generation of action potentials in a neuron was governed by its membrane potential $v^{j}(t)$ and a recovery current $u^{j}(t)$, as

$$
\begin{aligned}
\tau_{c} \frac{d v^{j}}{d t} & =k\left(v^{j}-v_{r}\right)\left(v^{j}-v_{t}\right)-u^{j}+I^{j}+\eta, \\
\tau_{a} \frac{d u^{j}}{d t} & =b\left(v^{j}-v_{r}\right)-u^{j}, \\
\text { If } v^{j} \geqslant v_{p} & \longrightarrow\left\{\begin{array}{l}
v^{j} \longleftarrow v_{c} \\
u^{j} \longleftarrow u^{j}+d,
\end{array}\right.
\end{aligned}
$$

where $\tau_{c}$ is the leaky capacitance; $v_{r}$ and $v_{t}$ are the resting and threshold potentials, respectively; $I^{j}$ is the synaptic current the $j$ th neuron received from the rest of the network; $\eta$ is a noise

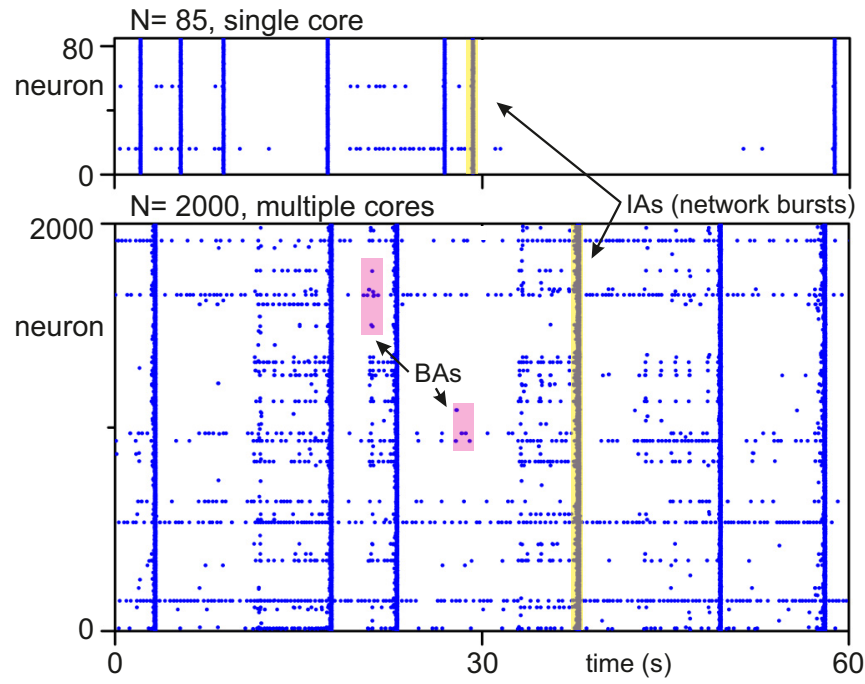

FIG. 4. Spontaneous activity in simulated networks. The plots show representative raster plots for a small network ( $N=85$ neurons, top) and a large one $(N=2000$, bottom). Blue dots are neuronal activations. Systemwide coherent events are ignition avalanches (IAs, yellow boxes) and shape network bursts. Correlated activations that do not lead to a coherent event in the whole culture are background avalanches (BAs, pink boxes).

term; $\tau_{a}$ is the main time scale of the inhibitory currents; and $b$ is related to the sensitivity of the neurons to subthreshold fluctuations. Also, $v_{p}$ is the value of the membrane potential at which a synaptic pulse is emitted; $v_{c}$ is the reset value of the membrane potential after spike emission; and $d$ accounts for the adaptation and recovery of the neurons.

Finally, for the synaptic dynamics, we considered that the generation of a synaptic pulse by the $i$ th neuron at a time $t_{m}$ induced a postsynaptic current in the $j$ th neuron. Thus, the total synaptic current received by the $j$ neuron was given by

$$
\begin{aligned}
I_{j}(t) & =\sum_{i=1}^{N} \sum_{t_{m}<t} A_{i j} E_{i}\left(t, t_{m}\right), \\
E_{i}\left(t, t_{m}\right) & =g_{A} D_{i}\left(t_{m}\right) \exp \left(-\frac{t-t_{m}}{\tau_{A}}\right) \Theta\left(t-t_{m}\right), \\
\frac{d D_{i}}{d t} & =\frac{1-D_{i}}{\tau_{D}}-(1-\beta) D_{i} \delta\left(t-t_{m}\right),
\end{aligned}
$$

where $A_{i j}$ is the connectivity matrix of the network, and $E_{i}\left(t, t_{m}\right)$ is the current induced by the $i$ th neuron at a time $t$ as a result of the spike generated at time $t_{m} ; g_{A}$ and $\tau_{A}$ are the strength and decay time of the synaptic current, respectively; $D_{i}(t)$ is the depression term that describes the efficacy of the neuron presynaptic terminals [46,47]. It has a resting value of 1 and relaxes exponentially with a decay time of $\tau_{D}$. Also, $\beta$ is a coefficient related to the loss in efficiency that occurs whenever a synaptic pulse is generated.

The in silico cultures were simulated along $1 \mathrm{~h}$, and the data were analyzed to quantify the spatiotemporal structure of activity avalanches in the networks (see Fig. 4 for an example of the time series of the bursts obtained in the simulations). Table II summarizes the parameters used in the construction of the numerical model. 
TABLE II. Values for the structural and dynamical parameters of the in silico neuronal cultures. AMPA stands for $\alpha$-amino-3-hydroxy5-methyl-4-isoxazolepropionic acid.

\begin{tabular}{|c|c|}
\hline \multicolumn{2}{|c|}{ Culture parameters } \\
\hline Density & $\rho=125$ neurons $/ \mathrm{mm}^{2}$ \\
\hline \multicolumn{2}{|l|}{ Morphology parameters } \\
\hline Diameter of the soma & $\phi_{s}=15 \mu \mathrm{m}$ \\
\hline Dendritic tree radius & $\mu_{d}=300 \mu \mathrm{m}, \sigma_{d}=40 \mu \mathrm{m}$ \\
\hline Axon length & $\sigma_{l}=800 \mu \mathrm{m}$ \\
\hline Axon cutoff length & $l_{\text {cutoff }}=1500 \mu \mathrm{m}$ \\
\hline Segment length & $\Delta l=10 \mu \mathrm{m}$ \\
\hline Angle between segments & $\sigma_{\theta}=0.1 \mathrm{rad}$ \\
\hline \multicolumn{2}{|l|}{ Soma parameters } \\
\hline Resting membrane potential & $v_{r}=-60 \mathrm{mV}$ \\
\hline Threshold membrane potential & $v_{t}=-45 \mathrm{mV}$ \\
\hline Peak membrane potential & $v_{p}=35 \mathrm{mV}$ \\
\hline Reset membrane potential & $v_{c}=-50 \mathrm{mV}$ \\
\hline Time scale of the inhibitory current & $\tau_{a}=50 \mathrm{~ms}$ \\
\hline Leaky capacitance & $\begin{array}{c}\tau_{c}=50 \mathrm{~ms} \\
k=0.5 \mathrm{mV}^{-1}\end{array}$ \\
\hline Sensitivity to subthreshold fluctuations & $b=0.5$ \\
\hline Adaptation and recovery parameter & $d=50 \mathrm{mV}$ \\
\hline \multicolumn{2}{|l|}{ Synapse parameters } \\
\hline Depression recovery time & $\tau_{D}=1000 \mathrm{~ms}$ \\
\hline Depression decay & $\beta=0.8$ \\
\hline AMPA current strength & $g_{A}=50 \mathrm{mV}$ \\
\hline AMPA current decay time & $\tau_{A}=5 \mathrm{~ms}$ \\
\hline \multicolumn{2}{|l|}{ Noise parameters } \\
\hline White noise strength & $g_{s}=300 \mathrm{mV}^{2} \mathrm{~ms}$ \\
\hline White noise autocorrelation & $\left\langle\eta(t) \eta\left(t^{\prime}\right)\right\rangle=2 g_{s} \delta\left(t-t^{\prime}\right)$ \\
\hline Shot noise frequency & $\lambda=0.01-0.05 \mathrm{~ms}^{-1}$ \\
\hline Shot noise strength (minis) & $g_{m}=10 \mathrm{mV}$ \\
\hline Shot noise decay time & $\tau_{m}=\tau_{A}$ \\
\hline \multicolumn{2}{|l|}{ Simulation parameters } \\
\hline Algorithm & Runge-Kutta second order \\
\hline Time step & $\delta t=0.01 \mathrm{~ms}$ \\
\hline Termalization time & $t_{\mathrm{term}}=10 \mathrm{~s}$ \\
\hline Integration time & $t_{\mathrm{int}}=1 \mathrm{~h}$ \\
\hline Simulation time & $t_{\mathrm{sim}}=t_{\mathrm{term}}+t_{\mathrm{int}}$ \\
\hline Typical run time & $T_{\text {ex }} \lesssim 3 \mathrm{~d}$ \\
\hline
\end{tabular}

\section{A. Dynamic amplification and avalanches}

As a first exploration, we considered networks with the same number of neurons as in a core, i.e., with $N=N_{c}=$ 85. As shown in Fig. 5(a) for a representative network, the dynamic profile (color dots and their statistical average) was qualitatively similar to the one predicted by the model [Fig. 2(a)]. A remarkable difference, however, was that the simulated profile was much more symmetric in shape than the theoretical one. This difference is grounded on two factors, namely, the presence of recurrent activity and the existence of burst-termination mechanisms. The former is associated with metric correlations and translates into the high activity levels observed by $n \simeq 30-50$. The latter is associated with neuronal hyperpolarization and synaptic depression and leads to an abrupt decline of activity by $n \simeq 70-80$.

We note that, to compute the dynamical profile in simulations, a rolling average with a time window $w$ was implemented on the original raster plots as a smoothing
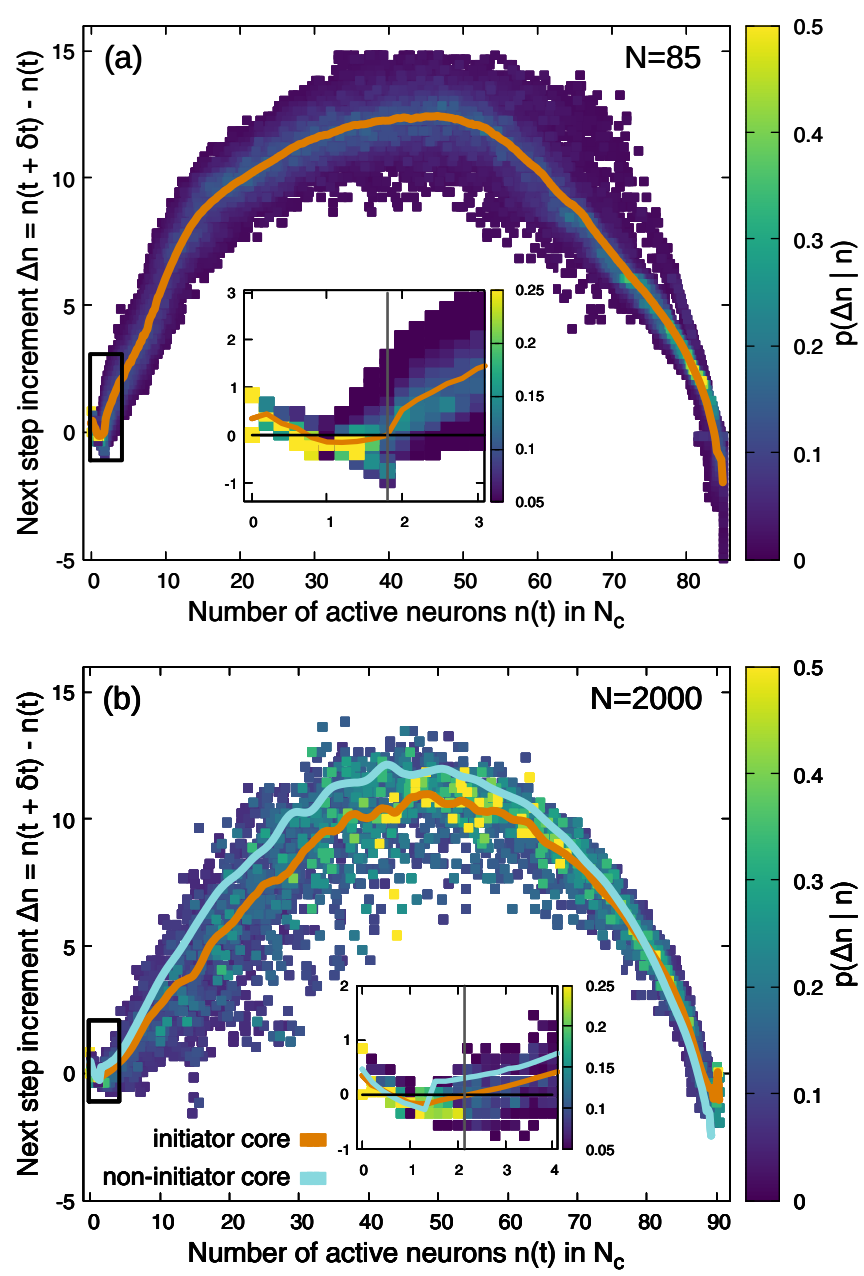

FIG. 5. Dynamic profile in representative simulated neuronal networks. (a) An isolated network core with $N=N_{c}=85$ neurons placed on an area of $\phi=600 \mu \mathrm{m}$ in diameter. Each colored dot represents a step in an avalanche process, with the color scale indicating its observed occurrence $p$. The orange curve represents the statistical average of the data points $\langle\Delta n\rangle(n)=\sum_{\Delta n} \Delta n p(\Delta n \mid n)$. The inset shows a detail of the avalanche process for low $n$ and the determination of the threshold $n_{\text {th }} \simeq 2$ that separates background avalanches (BAs) from ignition avalanches (IAs) (vertical gray line), obtained as the intercept of the increasing orange curve and the abscissa axis. (b) Equivalent analysis for cores integrated in a large network of $N=2000$ neurons. Two populations are considered, a first one with $N_{c}=91$ that leads burst formation (initiation core, color dots and orange curve) and a second one with the same number of neurons that does not initiate activity (nonintiation area, cyan curve). Both curves are qualitatively similar since any generated burst propagates throughout the entire network. In all panels, statistics is based on about 5000 avalanches in 10-h-long simulations.

process. For this reason, the number of active neurons in $N_{c}$, $n$, and the next-step increment $\Delta n$ are not limited to integers. Additionally, a bin of width $\Delta n_{\text {bin }}=1 /(2 w+1)$ was used to compute the occurrence $p(\Delta n \mid n)$, which was defined as the probability that a core with a given $n$ increased its activity by $\Delta n$. This $p(\Delta n \mid n)$ was determined by counting the number of events in which $n \rightarrow n+\Delta n$ and normalizing by the total observed $\Delta n, \sum_{\Delta n}(n \rightarrow n+\Delta n)$. 
The simulations procured a threshold $n_{\text {th }} \simeq 2$ and a maximum next-step increment $\Delta n^{\max } \simeq 15$, both smaller than the model predictions ( $n_{\mathrm{th}} \simeq 4.7$ and $\Delta n^{\text {max }} \simeq 43$, respectively). We ascribe these differences to the characteristics of the Izhikevich model employed and the topology of the $N_{c}$ core, which may be more structured than the random graph assumed by the model. These aspects are discussed later, but the good qualitative agreement between model and simulations indicates that the former, despite its approximations, captures well the amplification mechanism and the presence of both BAs and IAs.

As a second exploration, we simulated more realistic neuronal networks of $N=2000$ neurons placed on a circular area of $\phi=3 \mathrm{~mm}$ in diameter. We then inspected the dynamics in the network and searched for the strongest initiator core, i.e., the area where bursts initiated more frequently. A circular area of radius $r_{c}=300 \mu \mathrm{m}$, centered in this core, was next set and its 91 containing neurons monitored in detail. As shown in Fig. 5(b) (orange curve), the dynamical profile for this integrated core is qualitatively similar to the isolated one, indicating that a burst initiation area in a large network can be effectively viewed as a neighborhood of highly interacting neurons.

The same core radius of $r_{c}=300 \mu \mathrm{m}$ was used to compare the simulations with the theoretical predictions. This was considered valid because short-scale connectivity (within the $N_{c}$ core) approached a random graph. However, since the numerical networks were generated as biologically realistic spatial graphs, the resulting cores exhibited variability in their internal connectivity, spatial extent, and the way they interconnected to one another. This variability favored topological amplification within the core, i.e., enhanced the capacity of the core to generate a burst, but also provided intricate large-scale connectivity correlations that ultimately led few initiation cores to dominate the dynamics. Indeed, of all $N / N_{c}=2000 / 85 \simeq 23$ potential cores, only $3-5$ concentrated all bursts, shaping truly special initiation areas or focusing points, as in Orlandi et al. [30].

In Fig. 5, a difference worth noting between the isolated $\left(N \simeq N_{c}\right)$ and integrated $\left(N \gg N_{c}\right)$ cores is the strong variability in avalanche occurrence for the latter, reflected by the abundant color patterning in Fig. 5(b). We ascribe this difference to the strong dynamic interactions between the integrated core and the rest of the network and with bursts or avalanches originating in other cores crossing the monitored one.

To shed light on this aspect, we compared the dynamic profiles of this integrated core with an area in the network that did not initiate activity but contained the same 91 monitored neurons. The resulting curve is shown in cyan in Fig. 5(b). The curve shows a sharper increase than the core one (orange) since any burst generated in the network, together with local BAs, would contribute to activity in the monitored region. Additionally, the threshold that separates BAs from IAs appears at lower $n$ and changes sharply because many neurons suddenly become active when a burst crosses the monitored area. However, despite these differences, the core and noncore dynamic profiles exhibit a similar trend, which indicates that all neurons have a similar capacity for activity initiation and propagation. Thus, although connectivity may be heterogeneous at a local scale, it is quite uniform at larger scales. This is expected since neurons are distributed homogeneously and all form connections with identical rules.

\section{B. Occurrence and resilience of bursts in large networks}

After exploring the dynamics of both isolated and integrated cores, we proceeded to study the temporal occurrence of bursting dynamics. We considered the scenario of more realistic networks with $N=2000$ neurons and determined the characteristic IBI in the 1-h-long simulations, averaging over 50 network realizations. We obtained $\langle\mathrm{IBI}\rangle=14 \pm 8 \mathrm{~s}$, a value that is in agreement with the theoretical prediction of Eq. (20).

To complete the results, as well as to better characterize the parameters governing bursting formation, we studied the dependence of IBIs on the number of neurons in the network and the sensitivity of bursting phenomena to the loss of neurons.

\section{Dependence of bursting on network size}

The dependence of bursting occurrence on the number of neurons $N$ could be predicted through Eq. (20). Indeed, by evaluating $\mathcal{P}\left(n_{\mathrm{th}}, \omega_{0} \Delta t N_{c}\right)$ for the values $n_{\mathrm{th}}=4.7$ neurons, $\omega_{0}=0.1 \mathrm{~Hz}, \Delta t=20 \mathrm{~ms}$, and $N_{c}=85$ neurons, we observed that $\mathcal{P}\left(n_{\mathrm{th}}, \omega_{0} \Delta t N_{c}\right)=1-\delta$ with $\delta \ll 1$, and therefore,

$$
\left[\mathcal{P}\left(n_{\mathrm{th}}, \omega_{0} \Delta t N_{c}\right)\right]^{\frac{N}{N_{C}}}=(1-\delta)^{\frac{N}{N_{C}}} \approx 1-\frac{N}{N_{c}} \delta,
$$

leading to

$$
\langle\mathrm{IBI}\rangle \simeq \tau_{\mathrm{rec}}+\frac{\Delta t}{1-\mathcal{P}\left(n_{\mathrm{th}}, \omega_{0} \Delta t N_{c}\right)} \frac{N_{c}}{N},
$$

which indicates that $\langle\mathrm{IBI}\rangle \sim N^{-1}$.

To verify this scaling, we simulated networks with gradually higher numbers of neurons $N$ that covered about two orders of magnitude, from $N=100$ to 2000, and a total of six population sizes. Bigger networks could not be explored because of the prohibitive duration of the simulations.

Representative IBI distributions for the smallest and largest networks are shown in Fig. 6(a). The smallest network shows a large average IBI of about $\langle\mathrm{IBI}\rangle-\tau_{\text {rec }} \simeq 90 \mathrm{~s}$, whose distribution is markedly exponential. We ascribe this behavior to the existence of a single initiation core in the network, and that leads to a strong correlation between two consecutive bursts since the capacity for a new burst to emerge strongly depends on the full termination of the previous one. Conversely, the biggest network exhibits a substantially smaller average IBI of about $\langle\mathrm{IBI}\rangle-\tau_{\mathrm{rec}} \simeq 6 \mathrm{~s}$ and a characteristic Poissonian distribution that is indicative of a random generation of bursts across the network. Indeed, the presence of multiple cores erases the correlation between burst initiation times, except for the global constraint that a burst cannot occur at time scales shorter than $\tau_{\text {rec }}$.

The scaling of IBI on $N^{-1}$ predicted by Eq. (24) is shown in Fig. 6(b). Despite the strong variability among realizations, the data followed well a power law with exponent $-1.16 \pm 0.21$, compatible with the theoretical prediction. It is important to note that the smallest networks (with $N=100$ or 200 neurons) exhibited strong variations in the IBI. This was caused, first, by the fact that these network contained one or two cores and often were at the limit of showing bursting 

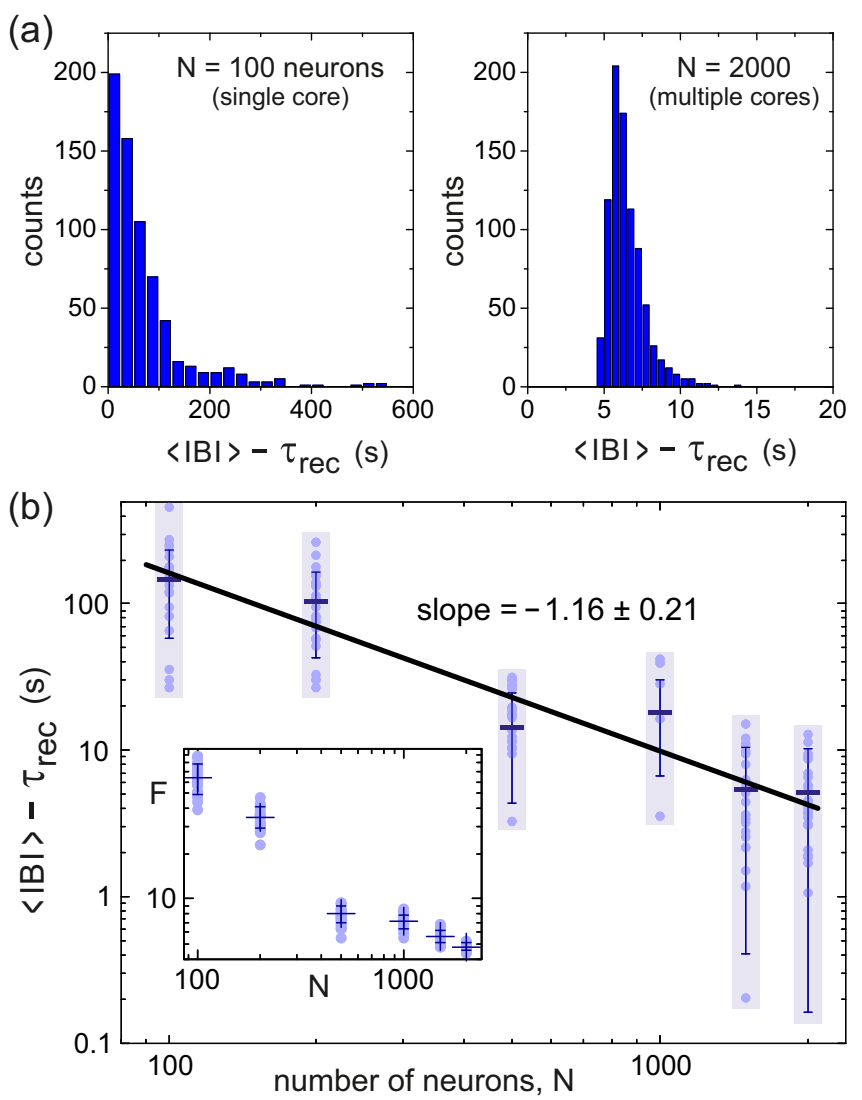

FIG. 6. Bursting statistics and dependence on network size. (a) Interburst interval (IBI) distributions for a single numerical realization of a small (left) and a large network (right). The former has an exponential-like shape and large IBIs. The latter is Poissonian with small IBIs. (b) Main plot: Power law dependence of $\langle I B I\rangle-\tau_{\text {rec }}$ on $N$. Statistics for each $N$ are based on 25 network realizations (light blue dots). Color boxes show the extent of the distribution. Thick and thin horizontal lines within each box show, respectively, the mean and standard deviation. The black line is a linear fit on a log-log scale. The uncertainty in the fit indicates a statistical confidence level of $95 \%$. Inset: Fano factor $F$ as a function of $N$, showing the tendency toward a more regular bursting as the size of the system grows.

activity. This is clear, for instance, in the top raster plot of Fig. 4, in which there is a long period of silence in the network. Second, the capacity of these small networks to activate was sensitive to their connectivity blueprint in a given realization, combined with dynamics that was strongly affected by finite size effects. The variability in the IBIs is captured by the Fano factor $F$, computed as $F=\sigma_{\mathrm{IBI}}^{2} / \mu_{\mathrm{IBI}}$, with $\sigma_{\mathrm{IBI}}^{2}$ the variance of $\langle\mathrm{IBI}\rangle-\tau_{\text {rec }}$ and $\mu_{\mathrm{IBI}}$ its standard deviation. The dependence of $F$ on $N$ is shown in the inset of Fig. 6(b), with $F$ decreasing by a factor of 10 from the smallest to the biggest networks. This additional analysis emphasizes the strong increase in bursting regularity as network size grows.

\section{Resilience of burst initiation to the loss of neurons}

Here, we considered networks with $N=2000$ neurons and applied consecutive rounds of directed attacks, defined as the removal of all the neurons in the network that initiated $>10 \%$ of the IAs and, thus, played a central role in burst initia- tion. After each round, the IBI of the damaged culture was measured and the procedure repeated. The results for eight different culture realizations and their average is shown in Fig. 7(a). For clarity, the IBI in each attack round is scaled relative to the initial, unperturbed condition $\left(\mathrm{IBI}_{0}\right)$. As a general trend, the IBI of the networks gradually grew with the number of attacks and increased by about two orders of magnitude relative to the initial condition.

We note that the bursting phenomenon was maintained despite the attack on neurons. This indicates that there is always a sufficient amplification of activity, either by topology or noise, for new neurons to take the leadership in avalanche generation. Indeed, $\sim 30 \%$ of the neurons in the network were removed after the third round of attack, but the remaining ones were still capable of generating activity. As illustrated in Fig. 7(b), the foci of initiation varied after each attack, reflecting the intricate interplay between connectivity, neuronal activity, and noise that determines burst initiation areas, in which both local (e.g., noise) and nonlocal (topology) amplification mechanisms are central. We observed that $\sim 50 \%$ of the neurons had to be removed to fully silence the network. This reflects a strong resilience of bursting to network damage and illustrates the difficulty to control or suppress it by just focal damage.

\section{DISCUSSION}

The emergence of extreme events appears recurrently in the dynamics of social and natural systems at different scales. This ubiquity is grounded on the existence of microscopic amplification mechanisms based on nonlinear elements coupled and mediated by complex patterns of connectivity and noise. Here, centering on the amplification of activity in neural networks, we have presented a general framework whose main ingredients can be used as a skeleton to model a variety of extreme events that appear in complex systems.

Our framework was described in the context of neuronal networks since they represent a prominent paradigm of the importance of connectivity and noise in governing amplification phenomena. We showed that the ignition of a core of neurons as small as $N_{c} \simeq 85$ neurons sufficed to initiate a large-scale activity avalanche that extended throughout the entire network. We used biologically realistic numerical simulations of neuronal networks to validate the predictions of the model. Simulations were in good agreement with the theoretical behavior, mimicking neatly the dynamic profile and portraying a clear threshold between BAs and IAs, with the latter ultimately shaping a network burst. Simulations, however, showed lower values of next-step increment $\Delta n$ in the dynamic profile, typically by a factor of three, and a lower threshold separating BAs from IAs of $n_{\mathrm{th}}^{\mathrm{sim}} \simeq 2$ (model, $n_{\mathrm{th}}^{\text {mod }} \simeq 4.7$ ). We associate these differences to two central aspects of the simulations, namely, a realistic dynamic description for neurons and synapses and a nonrandom construction of the network connectivity.

For the first aspect, simulations used the integrate-andfire Izhikevich model with short-term synaptic depression to describe the behavior of firing neurons, which adds a higher degree of biophysical accuracy than the theoretical description. Among other features, the model considers the 


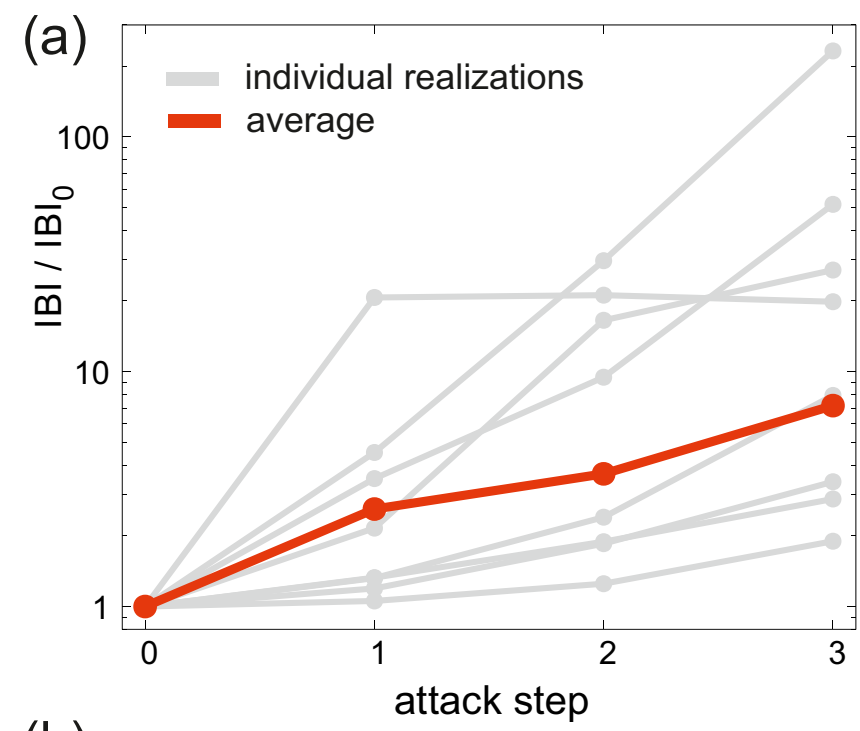

(b)
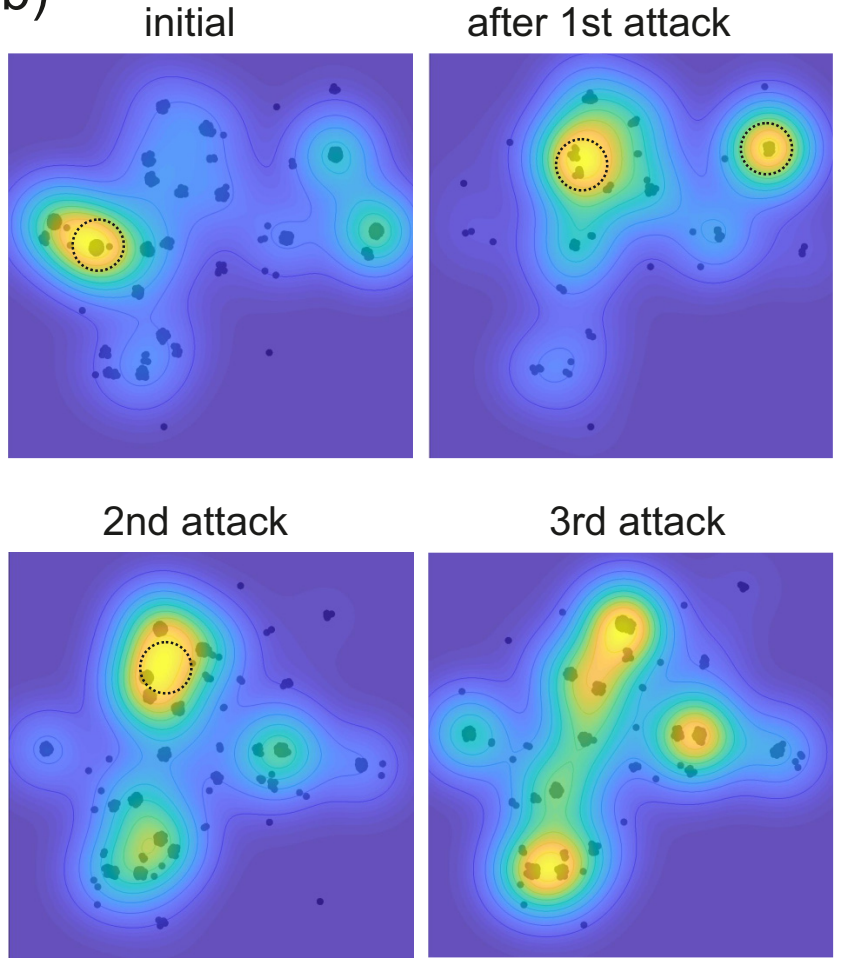

FIG. 7. Resilience of burst initiation. (a) Evolution of the average interburst interval (IBI) for eight cultures (gray) with $N=2000$ neurons for three progressive directed attacks on the most active neurons. The attack consisted of the deletion of all neurons that initiated $>10 \%$ of the avalanches in a 1-h-long simulation. For each realization, the IBI is scaled relative to the unperturbed initial condition. On average (red), IBI increases with the degree of damage. (b) Observed burst initiation points (black dots) and corresponding probability density function of spatial burst initiation (blue-yellow color pattern). The brighter the color, the higher the probability of a burst to occur in a particular region of the network. The circular black outlines show the areas that experienced the strongest deletion of neurons upon attack. The position of the neurons is not shown for clarity. exponential decay of synaptic efficiency due to the depletion of neurotransmitters, a characteristic that reduces the degree of spiking bombardment among neurons and, thus, lowers $\Delta n$. For the second aspect, simulations considered a biologically realistic formation of neuronal circuits, with neurons placed on a two-dimensional substrate and with distancedependent connectivity probability. These features shaped a network with inherited spatial correlations that shaped loops and heterogeneity in the connectivity. Only at the scales of the dendritic tree, the connectivity approaches a random graph [32,45], as Eq. (17) of the theoretical description assumes. We argue that, in the simulations, the presence of spatial correlations further facilitates activity amplification, particularly at distances beyond the core itself, increasing the probability to observe IAs and thus lowering $n_{\text {th }}$.

The model and numerical simulations show that a network connectivity given by a random graph is sufficient to ignite a burst. This is because noise amplification provides the necessary drive for activity to grow beyond BAs and activate the entire network. Topological amplification only participated in a minor way, as discussed above. However, the capacity of topology to amplify activity and govern burst formation is interesting for two reasons. First, because by shaping specific connectivity blueprints, such as feedforward and feedbackward loops, amplification can substantially increase and become as important as noise [30]. Second, because by appropriately tuning network connectivity, it is possible to shape bursts with different sizes or rich spatiotemporal structure and not only the extreme whole-network activation seen in our study. Tuning network connectivity is a fascinating problem that we explored both in experiments and simulations. In experiments, cultures with rich dynamics were shaped as either interconnected modules [48] or highly dense neuronal assemblies [49-51]. For simulations, we investigated the impact of strong spatial heterogeneity in the distribution of neurons [49] or the incorporation of specific geometries for connectivity guidance [37].

The theoretical model that we introduced does not consider inhibition, an important and intrinsic trait of neuronal circuits. Its role, however, is complex. In the context of homogeneous cultures, inhibition can be viewed as an effective reduction of excitation [33,41], leading to a lower connectivity $k$ and thus a reduced capacity for the system to reach the threshold for activating the core. In this context, inhibition should be expected to reduce the number of bursting events observed in the network. However, inhibition also acts as an additional mechanism for burst termination, preventing the pools of neurotransmitters from becoming totally depleted, thus facilitating a faster recovery time. Experimental evidence [30,41] shows that, in the presence of inhibition, bursts do indeed become weaker, and the IBI decreases by a factor of $2-5$.

Regarding the distribution of avalanche sizes, the bursts observed in our model recruit the whole system, i.e., all neurons become active when an IA occurs (Fig. 4). This is due to the lack of inhibition and the combination of a homogeneous distribution of neurons with strong metric connectivity correlations. This effect can be understood as a hybrid percolation transition (HPT) akin to the quorum, bootstrap percolation, or threshold models [36,52,53]. However, the novel inclusion of noise within our study smooths out the sharp first-order 
transition of the HPT and gives rise instead to a characteristic first-passage time, which in the context of our neuronal network would correspond to the bursting time [38]. Moreover, whereas the IAs extend to the entire network, the BAs do not. One could expect a broad distribution of BA sizes, for instance, in the form of a power-law, and that could indicate a critical behavior. However, power-law-distributed BAs were not observed in our system due to the presence of spontaneous activations, which cause a temporal superposition of activity cascades and eventual merging of avalanches. This superposition ultimately leads to a mixture of classical and directed percolation behaviors with mixed exponents [54], whose analysis is beyond the scope of this paper.

A remarkable aspect of our study is the robustness of burst initiation, in which the deletion of central nodes alters but does not suppress amplification mechanisms and burst formation. The exploration of network behavior through targeted attack indicates that the network must be severely damaged for bursting behavior to disappear. From a neuroscience perspective, this can be viewed as both positive and negative: positive because such a resilience ensures that neuronal circuits will always maintain structured collective activity despite moderate physical damage or perturbations. Even if the IBI increases upon insult, the capacity of the network to exhibit spontaneous activity may be sufficient to turn on homeostatic response mechanisms to damage and gradually return the network to an optimal set point, as observed in experiments in neuronal cultures $[55,56]$. It is negative because pathologically excessive bursting may be difficult to control or fully suppress. Additionally, in a more general complex system perspective, the resilience of bursting may indicate that extreme events are most likely impossible to eliminate and that efforts must be put into understanding not only the major actors at play and their interrelation, but also to predict as best as possible the characteristic time scales of extreme events.

The proposed model can be linked to the general problem of extreme events dynamics. Indeed, two different mechanisms contribute to the behavior reported here. On the one hand, the bursting behavior is due to the noise amplification on a single or a few nodes in the network, and on the other hand, heterogeneity in the connectivity determines the location where the bursting activity starts in the network. Similar dynamics can be observed in networks of excitable systems performing relaxation oscillations when isolated $[16,26]$.

Using FitzHugh-Nagumo or Morris-Lecar units on networks with different topologies, it has been shown that several spatiotemporal patterns can occur which are comparable with the findings reported here [16,26,57]. For low connectivity, one can find small-amplitude oscillations leading to localized patches of nodes that exhibit a synchronized excitation, which dies out without propagation through the network. Compared with the model studied here, this behavior can be interpreted as the equivalent of a BA. When a certain critical number of nodes forms a simultaneous excitation, this cluster of nodes can initiate a synchronized excitation of a large part of the whole network, called an extreme event in Ref. [16], and which could be interpreted as an IA in the sense of this paper.

These two types of behavior (BAs and IAs) occur already for a heterogeneous network with respect to the dynamics on the nodes but with a homogeneous coupling topology, realized as a fully coupled network. By considering heterogeneity in the connectivity by employing a small-world topology, additional traveling waves of excitation can be observed if the coupling is close to homogeneous, i.e., only a few long-range connections exist. For larger values of rewiring probability, which further emphasizes the heterogeneity in the connectivity, an irregular switching process between small-amplitude oscillations, traveling waves, and extreme events can occur. For even larger rewiring probability, the whole FitzHughNagumo network shows permanent global oscillations, which would be comparable with permanent bursting in the model studied here. A further common property of our model and FitzHugh-Nagumo's is that, with increasing network size, the mean time between bursts and extreme events, respectively, decreases.

Similar findings have been reported in networks of pulsecoupled oscillators [27,58] as well as networks of leaky integrate-and-fire models [8,59]. In the first model system [27], transient patterns of synchrony occur, comparable with BAs for a large intermediate range of connectivity (more precisely rewiring probability in a small-world network), whereas large parts of the network synchronize for higher connectivity, resembling IAs. In the second model [59], such transient space-time patterns resulting from a learning mechanism are observable only in a critical point between replay and nonreplay dynamics. Again, this transient could be interpreted as a neuronal avalanche resembling a BA. As in this paper, the mechanism behind the formation of these avalanches in the different variants of integrate-and-fire models lies in the intricate interplay between noise and topology. The former is introduced as heterogeneity in the nodes or random stimuli, mimicking spontaneous activity of the neurons. The latter is either described as a varying rewiring probability [27] or realized as a result of a design of synaptic connections based on a spike-timing-dependent plasticity rule [59], respectively.

Considering all those commonalities, we can formulate the general properties a system should possess to show the reported BAs and IAs: (i) the network should be able to self-generate and self-terminate localized excitations for several nodes due to their heterogeneity, which could be either realized by a spread of frequencies of the isolated oscillatory nodes or the noise impact on excitable units; (ii) when only a small number of nodes is excited in a short time interval, only BAs can be formed, whereas for IAs, the first excitation has to comprise an overcritical number of nodes; and (iii) heterogeneity in the node dynamics, as well as in the connectivity among the nodes, determines where the IA starts.

Based on these general properties, we can identify several natural systems beyond neuroscience in which such dynamics can be expected. Two are of particular interest in the context of environment and health. The first one is the dynamics of harmful algal blooms (HABs) in the ocean. Blooms of specific harmful or even toxic species can be described in terms of an excitable system $[5,60]$ in which such a bloom can be triggered by fluctuations in temperature [61] or predators controlling the growth of the community of various phytoplankton species [5]. Different regions at the coast characterized by different physical, chemical, and biological properties — called hydrodynamic provinces [62]—are connected by different ocean currents such that the dynamics can 
be described as a network whose connectivity is determined by the hydrodynamic flows in the ocean. The propagation of HABs along the flow patterns could be regarded as one possible example for the formation of BAs and IAs [63]. The second one is the dynamics of transmissible diseases in which sudden and explosive outbreaks occur as a product of the spread of circulating pathogens $[6,64]$. These outbreaks, sometimes called superspreading events [65], are the result of the interconnection between humans forming social networks of correlated and densely connected clusters combined with the stochastic nature of the transmission of pathogens.

In conclusion, the model and phenomena reported here can be viewed in a more general context in terms of extreme events on networks. The necessary ingredients would be oscillatory dynamics on the nodes and a mutual coupling with different topologies. The oscillatory dynamics on the nodes could be either deterministic or stochastic, which would not be perfectly oscillatory but, as demonstrated here, sufficient to produce extreme events.

\section{ACKNOWLEDGMENTS}

This paper was supported by MINECO and FEDER funds through Projects No. MAT2015-69-777-REDT, No. FIS201678507-C2-2-P, No. FIS2017-87519-P, No. FIS2017-90782REDT (IBERSINC), and No. PID2019-108842GB-C21; by the Departamento de Industria e Innovación del Gobierno de Aragón y Fondo Social Europeo through Grant No. E36-17R (FENOL); and by the Generalitat de Catalunya through Grants No. 2014FI-B00721 and No. 2017-SGR-1061.

Lluís Hernández-Navarro and Sergio Faci-Lázaro contributed equally to this paper.
[1] A. Bunde, J. Kropp, and H.-J. Schellnhuber, The Science of Disaster (Springer, Berlin, Heidelberg, 2002).

[2] D. Sornette, Critical Phenomena in Natural Sciences (Springer, Berlin, Heidelberg, 2003).

[3] S. Albeverio, V. Jentsch, and H. Kantz (eds.) Extreme Events in Nature and Society, The Frontiers Collection (Springer, Berlin, 2006).

[4] D. M. Anderson, A. D. Cembella, and G. M. Hallegraeff, Progress in understanding harmful algal blooms: Paradigm shifts and new technologies for research, monitoring, and management, Annu. Rev. Mar. Sci. 4, 143 (2012).

[5] S. Chakraborty and U. Feudel, Harmful algal blooms: Combining excitability and competition, Theor. Ecol. 7, 221 (2014).

[6] R. Pastor-Satorras, C. Castellano, P. Van Mieghem, and A. Vespignani, Epidemic processes in complex networks, Rev. Mod. Phys. 87, 925 (2015).

[7] J. M. Beggs and D. Plenz, Neuronal avalanches in neocortical circuits, J. Neurosci. 23, 11167 (2003).

[8] S. Scarpetta, I. Apicella, L. Minati, and A. de Candia, Hysteresis, neural avalanches, and critical behavior near a first-order transition of a spiking neural network, Phys. Rev. E 97, 062305 (2018).

[9] K. Lehnertz, S. Bialonski, M.-T. Horstmann, D. Krug, A. Rothkegel, M. Staniek, and T. Wagner, Synchronization phenomena in human epileptic brain networks, J. Neurosci. Methods 183, 42 (2009).

[10] K. Dysthe, H. E. Krogstad, and P. Müller, Oceanic rogue waves, Annu. Rev. Fluid Mech. 40, 287 (2008).

[11] A. Pleskachevsky, S. Lehner, and W. Rosenthal, Storm observations by remote sensing and influences of gustiness on ocean waves and on generation of rogue waves, Ocean Dynam. 62, 1335 (2012).

[12] A. Chabchoub, N. P. Hoffmann, and N. Akhmediev, Rogue Wave Observation in a Water Wave Tank, Phys. Rev. Lett. 106, 204502 (2011).

[13] D. Solli, C. Ropers, P. Koonath, and B. Jalali, Optical rogue waves, Nature (London) 450, 1054 (2007).

[14] J. M. Dudley, F. Dias, M. Erkintalo, and G. Genty, Instabilities, breathers and rogue waves in optics, Nat. Photonics 8, 755 (2014).
[15] J. Zamora-Munt, B. Garbin, S. Barland, M. Giudici, Jose R. Rios Leite, C. Masoller, and J. R. Tredicce, Rogue waves in optically injected lasers: origin, predictability, and suppression, Phys. Rev. A 87, 035802 (2013).

[16] G. Ansmann, R. Karnatak, K. Lehnertz, and U. Feudel, Extreme events in excitable systems and mechanisms of their generation, Phys. Rev. E 88, 052911 (2013).

[17] J. A. Reinoso, J. Zamora-Munt, and C. Masoller, Extreme intensity pulses in a semiconductor laser with a short external cavity, Phys. Rev. E 87, 062913 (2013).

[18] R. Karnatak, G. Ansmann, U. Feudel, and K. Lehnertz, Route to extreme events in excitable systems, Phys. Rev. E 90, 022917 (2014).

[19] S. L. Kingston, K. Thamilmaran, P. Pal, U. Feudel, and S. K. Dana, Extreme events in the forced Liénard system, Phys. Rev. E 96, 052204 (2017).

[20] A. Mishra, S. Saha, M. Vigneshwaran, P. Pal, T. Kapitaniak, and S. K. Dana, Dragon-king-like extreme events in coupled bursting neurons, Phys. Rev. E 97, 062311 (2018).

[21] A. N. Pisarchik, R. Jaimes-Reátegui, R. Sevilla-Escoboza, G. Huerta-Cuellar, and M. Taki, Rogue Waves in a Multistable System, Phys. Rev. Lett. 107, 274101 (2011).

[22] A. N. Pisarchik, R. Jaimes-Reategui, R. Sevilla-Escoboza, and G. Huerta-Cuellar, Multistate intermittency and extreme pulses in a fiber laser, Phys. Rev. E 86, 056219 (2012).

[23] M. Onorato, S. Residori, U. Bortolozzo, A. Montina, and F. T. Arecchi, Rogue waves and their generating mechanisms in different physical contexts, Phys. Rep. 528, 47 (2013).

[24] N. Akhmediev et al., Roadmap on optical rogue waves and extreme events, J. Optics 18, 063001 (2016).

[25] J. M. Soto-Crespo, P. Grelu, and N. Akhmediev, Dissipative rogue waves: extreme pulses generated by passively modelocked lasers, Phys. Rev. E 84, 016604 (2011).

[26] G. Ansmann, K. Lehnertz, and U. Feudel, Self-Induced Switchings between Multiple Space-Time Patterns on Complex Networks of Excitable Units, Phys. Rev. X 6, 011030 (2016).

[27] A. Rothkegel and K. Lehnertz, Recurrent events of synchrony in complex networks of pulse-coupled oscillators, Eur. Phys. Lett. 95, 38001 (2011). 
[28] H. Kamioka, E. Maeda, Y. Jimbo, H. P. Robinson, and A. Kawana, Spontaneous periodic synchronized bursting during formation of mature patterns of connections in cortical cultures, Neurosci. Lett. 206, 109 (1996).

[29] D. A. Wagenaar, J. Pine, and S. M. Potter, An extremely rich repertoire of bursting patterns during the development of cortical cultures, BMC Neurosci. 7, 11 (2006).

[30] J. G. Orlandi, J. Soriano, E. Alvarez-Lacalle, S. Teller, and J. Casademunt, Noise focusing and the emergence of coherent activity in neuronal cultures, Nat. Phys. 9, 582 (2013).

[31] D. Lonardoni, H. Amin, S. Di Marco, A. Maccione, L. Berdondini, and T. Nieus, Recurrently connected and localized neuronal communities initiate coordinated spontaneous activity in neuronal networks, PLoS Comput. Biol. 13, e1005672 (2017).

[32] L. Hernández-Navarro, J. G. Orlandi, B. Cerruti, E. Vives, and J. Soriano, Dominance of Metric Correlations in TwoDimensional Neuronal Cultures Described through a Random Field Ising Model, Phys. Rev. Lett. 118, 208101 (2017).

[33] J. Soriano, M. R. Martínez, T. Tlusty, and E. Moses, Development of input connections in neural cultures, Proc. Natl. Acad. Sci. USA 105, 13758 (2008).

[34] I. Breskin, J. Soriano, E. Moses, and T. Tlusty, Percolation in Living Neural Networks, Phys. Rev. Lett. 97, 188102 (2006).

[35] O. Feinerman, A. Rotem, and E. Moses, Reliable neuronal logic devices from patterned hippocampal cultures, Nat. Phys. 4, 967 (2008).

[36] O. Cohen, A. Keselman, E. Moses, M. R. Martínez, J. Soriano, and T. Tlusty, Quorum percolation in living neural networks, Europhys. Lett. 89, 18008 (2010).

[37] A.-A. Ludl and J. Soriano, Impact of physical obstacles on the structural and effective connectivity of in silico neuronal circuits, Front. Comput. Neurosci. 14, 77 (2020).

[38] J. G. Orlandi and J. Casademunt, Stochastic quorum percolation and noise focusing in neuronal networks, Europhys. Lett. 133, 48002 (2021).

[39] D. B. Owen, A table of normal integrals, Commun. Stat. Simul. Comput. 9, 389 (1980).

[40] G. G. Turrigiano, K. R. Leslie, N. S. Desai, L. C. Rutherford, and S. B. Nelson, Activity-dependent scaling of quantal amplitude in neocortical neurons, Nature (London) 391, 892 (1998).

[41] E. Tibau, M. Valencia, and J. Soriano, Identification of neuronal network properties from the spectral analysis of calcium imaging signals in neuronal cultures, Frontiers Neural Circuits 7, 199 (2013).

[42] T. A. Gritsun, J. le Feber, and W. L. Rutten, Growth dynamics explain the development of spatiotemporal burst activity of young cultured neuronal networks in detail, PloS One 7, e43352 (2012).

[43] M. Bisio, A. Bosca, V. Pasquale, L. Berdondini, and M. Chiappalone, Emergence of bursting activity in connected neuronal sub-populations, PloS One 9, e107400 (2014).

[44] S. Faci-Lázaro, J. Soriano, and J. Gómez-Gardeñes, Impact of targeted attack on the spontaneous activity in spatial and biologically-inspired neuronal networks, Chaos 29, 083126 (2019).

[45] Q. Wen, A. Stepanyants, G. N. Elston, A. Y. Grosberg, and D. B. Chklovskii, Maximization of the connectivity repertoire as a statistical principle governing the shapes of dendritic arbors, Proc. Natl. Acad. Sci. USA 106, 12536 (2009).

[46] E. M. Izhikevich, Simple model of spiking neurons, IEEE Trans. Neural Networks 14, 1569 (2003).

[47] E. Alvarez-Lacalle and E. Moses, Slow and fast pulses in 1-D cultures of excitatory neurons, J. Comput. Neurosci. 26, 475 (2009).

[48] H. Yamamoto, S. Moriya, K. Ide, T. Hayakawa, H. Akima, S. Sato, S. Kubota, T. Tanii, M. Niwano, S. Teller, J. Soriano, and A. Hirano-Iwata, Impact of modular organization on dynamical richness in cortical networks, Sci. Adv. 4, eaau4914 (2018).

[49] E. Tibau, A. Ludl, S. Rüdiger, J. G. Orlandi, and J. Soriano, Neuronal spatial arrangement shapes effective connectivity traits of in vitro cortical networks, IEEE Trans. Network Sci. Eng. 7, 435 (2020).

[50] S. Teller, C. Granell, M. De Domenico, J. Soriano, S. Gómez, and A. Arenas, Emergence of assortative mixing between clusters of cultured neurons, PLoS Comput. Biol. 10, e1003796 (2014).

[51] S. Teller, I. B. Tahirbegi, M. Mir, J. Samitier, and J. Soriano, Magnetite-amyloid- $\beta$ deteriorates activity and functional organization in an in vitro model for Alzheimer's disease, Sci. Rep. 5, 17261 (2015).

[52] T. Tlusty and J.-P. Eckmann, Remarks on bootstrap percolation in metric networks, J. Phys. A: Math. Theor. 42, 205004 (2009).

[53] D. Lee, W. Choi, J. Kértesz, and B. Kahng, Universal mechanism for hybrid percolation transitions, Sci. Rep. 7, 1 (2017).

[54] D. J. Korchinski, J. G. Orlandi, S.-W. Son, and J. Davidsen, Criticality in spreading processes without time-scale separation and the critical brain hypothesis, arXiv:1908.08163 (2019).

[55] S. Teller, E. Estévez-Priego, C. Granell, D. Tornero, J. Andilla, O. E. Olarte, P. Loza-Alvarez, A. Arenas, and J. Soriano, Spontaneous functional recovery after focal damage in neuronal cultures, eNeuro 7, ENEURO.0254 (2020).

[56] E. Estévez-Priego, S. Teller, C. Granell, A. Arenas, and J. Soriano, Functional strengthening through synaptic scaling upon connectivity disruption in neuronal cultures, Network Neurosci. 4, 1 (2020).

[57] J. Lafranceschina and R. Wackerbauer, Impact of weak excitatory synapses on chaotic transients in a diffusively coupled morris-lecar neuronal network, Chaos 25, 013119 (2015).

[58] A. Rothkegel and K. Lehnertz, Irregular macroscopic dynamics due to chimera states in small-world networks of pulse-coupled oscillators, New J. Phys. 16, 055006 (2014).

[59] S. Scarpetta and A. de Candia, Neural avalanches at the critical point between replay and no-replay of spatiotemporal patterns, PLoS One 8, e64162 (2013).

[60] J. Truscott and J. Brindley, Ocean plankton populations as excitable media, Bull. Math. Biol. 56, 981 (1994).

[61] J. A. Freund, S. Mieruch, B. Scholze, K. Wiltshire, and U. Feudel, Bloom dynamics in a seasonally forced phytoplanktonzooplankton model: Trigger mechanisms and timing effects, Ecol. Complex. 3, 129 (2006).

[62] V. Rossi, E. Ser-Giacomi, C. Lopez, and E. Hernandez-Garcia, Hydrodynamic provinces and oceanic connectivity from a transport network help designing marine reserves, Geophys. Res. Lett. 41, 2883 (2014). 
[63] S. Bialonski, D. A. Caron, J. Schloen, U. Feudel, H. Kantz, and S. D. Moorthi, Phytoplankton dynamics in the Southern California Bight indicate a complex mixture of transport and biology, J. Plankton Res. 38, 1077 (2016).

[64] V. Colizza, A. Barrat, M. Barthelemy, and A. Vespignani, The role of the airline transportation network in the prediction and predictability of global epidemics, Proc. Nat. Acad. Sci. USA 103, 2015 (2006).

[65] D. Adam, P. Wu, J. Wong, E. H. Y. Lau, T.K. Tsang, S. Cauchemez, G. M. Leung, and B. J. Cowling, Clustering and superspreading potential of SARS-CoV-2 infections in Hong Kong, Nat. Med. 26, 1714 (2020). 